\title{
Myelin basic protein enhances axonal regeneration from neural progenitor cells
}

\author{
Zhengjian Yan ${ }^{1 \dagger}$, Lei Chu ${ }^{1 \dagger}$, Xiaojiong Jia ${ }^{2}$, Lu Lin² and Si Cheng ${ }^{1 *}$
}

\begin{abstract}
Introduction: Stem cell therapy using neural progenitor cells (NPCs) shows promise in mitigating the debilitating effects of spinal cord injury (SCI). Notably, myelin stimulates axonal regeneration from mammalian NPCs. This led us to hypothesize that myelin-associated proteins may contribute to axonal regeneration from NPCs.

Methods: We conducted an R-based bioinformatics analysis to identify key gene(s) that may participate in myelinassociated axonal regeneration from murine NPCs, which identified the serine protease myelin basic protein (Mbp). We employed E12 murine NPCs, E14 rat NPCs, and human iPSC-derived Day 1 NPCs (D1 hNPCs) with or without CRISPR/Cas9-mediated Mbp knockout in combination with rescue L1-70 overexpression, constitutively-active VP16PPARY2, or the PPARy agonist ciglitazone. A murine dorsal column crush model of SCl utilizing porous collagen-based scaffolding (PCS)-seeded murine NPCs with or without stable Mbp overexpression was used to assess locomotive recovery and axonal regeneration in vivo.

Results: Myelin promotes axonal outgrowth from NPCs in an Mbp-dependent manner and that Mbp's stimulatory effects on NPC neurite outgrowth are mediated by Mbp's production of L1-70. Furthermore, we determined that Mbp/L1-70's stimulatory effects on NPC neurite outgrowth are mediated by PPARY-based repression of neuron differentiation-associated gene expression and PPARY-based Erk1/2 activation. In vivo, PCS-seeded murine NPCs stably overexpressing Mbp significantly enhanced locomotive recovery and axonal regeneration in post-SCI mice.
\end{abstract}

Conclusions: We discovered that Mbp supports axonal regeneration from mammalian NPCs through the novel Mbp/L1 cam/Ppary signaling pathway. This study suggests that bioengineered, NPC-based interventions can promote axonal regeneration and functional recovery post-SCl.

Keywords: Spinal cord injury, Axonal regeneration, Neural progenitor, Myelin, Mbp

\section{Highlights}

- Bioinformatics identified Mbp as akey gene in myelin-associated NPC axonal regeneration

- Myelinpromotes axonal outgrowth from NPCs in an Mbp-dependent manner
- Mbp's stimulatory effects on NPC neurite outgrowth are mediated by Mbp's production of L1-70.

- Mbp/L1-70's stimulatory effects on NPC neurite outgrowth are mediated by PPAR .

- Mbp-overexpressing NPCs enhanced locomotive recovery and axonal regeneration in post-SCI mice.
*Correspondence: celine0323@126.com

${ }^{\dagger}$ Zhengjian Yan and Lei Chu contributed equally to this work

${ }^{1}$ Department of Orthopedics, the Second Affiliated Hospital

of Chongqing Medical University, No. 76 Linjiang Road, Yuzhong District, Chongqing 400010, China

Full list of author information is available at the end of the article

\section{Background}

Spinal cord injury (SCI) is characterized by the presence of a pathophysiological cascade induced through physical disruption of the spinal column [1]. Although SCI accounts for only $0.01 \%$ injury occurrence globally

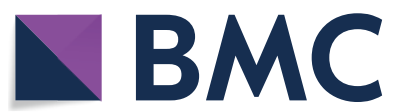

(c) The Author(s) 2021. This article is licensed under a Creative Commons Attribution 4.0 International License, which permits use, sharing, adaptation, distribution and reproduction in any medium or format, as long as you give appropriate credit to the original author(s) and the source, provide a link to the Creative Commons licence, and indicate if changes were made. The images or other third party material in this article are included in the article's Creative Commons licence, unless indicated otherwise in a credit line to the material. If material is not included in the article's Creative Commons licence and your intended use is not permitted by statutory regulation or exceeds the permitted use, you will need to obtain permission directly from the copyright holder. To view a copy of this licence, visit http://creativeco mmons.org/licenses/by/4.0/. The Creative Commons Public Domain Dedication waiver (http://creativecommons.org/publicdomain/ zero/1.0/) applies to the data made available in this article, unless otherwise stated in a credit line to the data. 
(700,000 diagnoses every year), SCI are physically debilitating and SCI per-patient care costs over $\$ 500,000$ in the first year and around $\$ 100,000$ annually for as long as the patient lives [2]. These factors result in large socioeconomic costs on healthcare systems globally.

Stem cell therapy has shown promise in helping to mitigate the effects of SCI by aiding surviving cell populations and regenerating neuronal and glial populations [3]. Thanks to recent progress in stem cell biology, it is now possible to produce terminally-differentiated homogeneous cell populations from totipotent or multipotent stem cell progenitors in vivo [3]. Neural progenitor cells (NPCs) grafted into lesions on rodent spinal cords result in the formation of new axons of up to $50 \mathrm{~mm}$ in length, a distance approximately ten thousand times greater than normal adult axons [4-7].

NPCs can form axons prior to myelination during central nervous system (CNS) embryonic development. Myelin-associated proteins-such as ephrins, myelinassociated glycoprotein (MAG), Nogo, netrin, and oligodendrocyte myelin glycoprotein (OMgp) - inhibit axonal regeneration from adult neurons [8]. Experimental interventions targeting these myelin-associated molecules have had success in increasing the growth of adult axons, resulting in hundreds of new axons in spinal cord white matter [8]. In contrast, myelin has been shown to stimulate axonal regeneration from mammalian NPCs [8]. This evidence that myelin can stimulate axonal regrowth from NPCs led us to hypothesize that myelin-associated proteins may contribute to axonal regeneration from NPCs.

In this study, we conducted an in silico bioinformatics analysis to identify key gene(s) that may participate in myelin-associated axonal regeneration from murine NPCs, which identified the serine protease myelin basic protein (Mbp). We also present in vitro and in vivo evidence that supports axonal regeneration from mammalian NPCs through the novel Mbp/L1 cell adhesion molecule (L1cam)/Ppary signaling pathway.

\section{Results}

\section{Bioinformatics analysis identifies Mbp as a key myelin-} induced transcript in NPCs

We conducted an R-based bioinformatics analysis on previously published RNA-seq data (GEO accession no. GSE98974 [8]) derived from murine E12 spinal cordderived NPCs (E12 mNPCs) that had been plated for $48 \mathrm{~h}$ on laminin (Lam), myelin (Mye), myelin+laminin (Mye +Lam), and PDL control (PDL Ctrl) substrate. These E12 mNPCs show similar neurite outgrowth profiles and responses to myelin stimulation as rat E14 spinal cord-derived NPCs (E14 rNPCs) and human iPSC-derived Day 1 NPCs (D1 hNPCs). We employed the R-based CemiTool package to ascertain the gene co-expression modules and pathways that differentiate the whole transcriptomes induced by the four substrates. We detected six distinct gene modules (Module [M]1-6) annotated by the mouse Reactome database (Fig. 1a). Based on the normalized enrichment scores (NES), four modules-M1, M2, M4, and M6 were enriched in the Mye-treated groups as compared to the non-Mye groups (Fig. 1a). Notably, the network diagrams of the four modules identified Mbp as a M6 hub gene (Fig. 1b, Additional file 1: Fig. S1A-D). GSEA revealed that M1 was enriched for various neurotransmitter release pathways, M2 was enriched for various protein folding and transport pathways, M4 was enriched for various transcriptional and translational pathways, and M6 was enriched for various FGFR signaling pathways (Additional file 1: Fig. S2A-D). DEG analysis revealed 6 upregulated and 33 downregulated genes in response to Mye substrate (Fig. 1d).

In order to specifically identify myelin-induced transcripts, we conducted a Venn analysis of upregulated DEGs from four comparisons: Mye vs. PDL Ctrl, Mye vs. Lam, Mye+Lam vs. PDL Ctrl, and Mye+Lam vs. Lam (Fig. 1e). We found four myelin-induced transcripts that were common across all comparisons: Plekhb1, S100b, Mbp, and Prph. Of these four transcripts, only Mbp was a member of an aforementioned gene module (i.e., M6). Therefore, we selected Mbp as a key myelin-induced transcript in NPCs for further investigation.

In order to validate our in silico findings, we employed qPCR and immunoblotting to measure myelin-induced Mbp mRNA and protein upregulation, respectively, in E12 mNPCs after a 48-h in vitro culture on myelin substrate (Fig. 1f, g). Notably, immunoblotting using a panMbp antibody recognized three major bands $(14,18$, and $21 \mathrm{kDa}$ ) for the Mbp protein (Fig. 1g). Moreover, qPCR and immunoblotting were performed to dynamically track Mbp mRNA and protein expression in maturing mNPCs (i.e., E12 to E17). The myelin-mediated increases in Mbp mRNA and protein levels gradually reduced on mNPC maturation from E12 to E17 (Fig. 1h, i).

\section{Myelin stimulates axonal outgrowth from NPCs in a mbp- dependent manner}

Three mammalian NPC models-E12 mNPCs, E14 rNPCs, or human iPSC-derived D1 hNPCs were utilized to better understand the mechanism(s) behind any Mbp-mediated effects on myelin-induced NPC dendrite regeneration. The selection of these particular NPC models was based on previous research on NPC axonal regeneration [8]. These NPCs, with or without CRISPR/ Cas9-mediated $M b p$ knockout, were plated on Mye or PDL Ctrl substrates for $48 \mathrm{~h}$. Western blotting validation of $M b p$ knockout using a pan-Mbp antibody recognized three major bands $(14,18$, and $21 \mathrm{kDa})$ in WT NPCs, 
a

M6

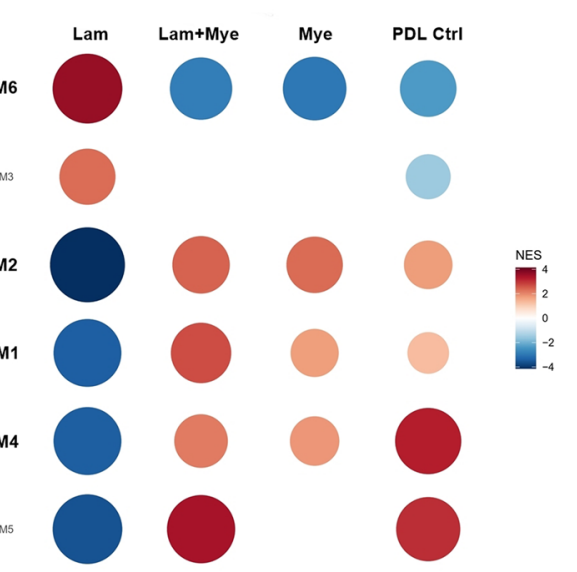

b

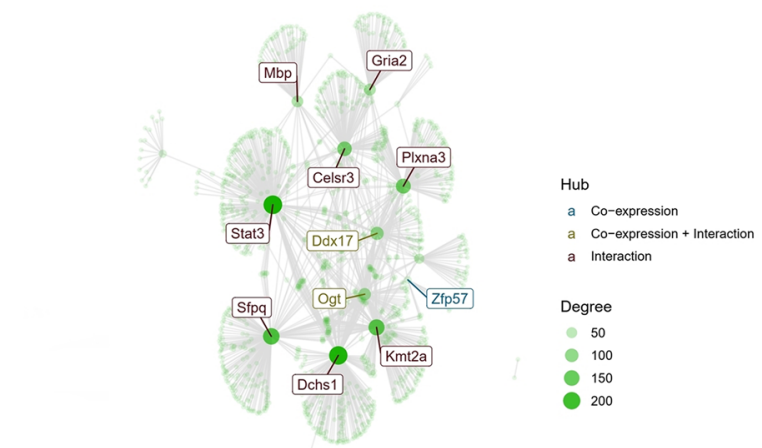

d

e

C

Dysregulated genes ordered by fold-change
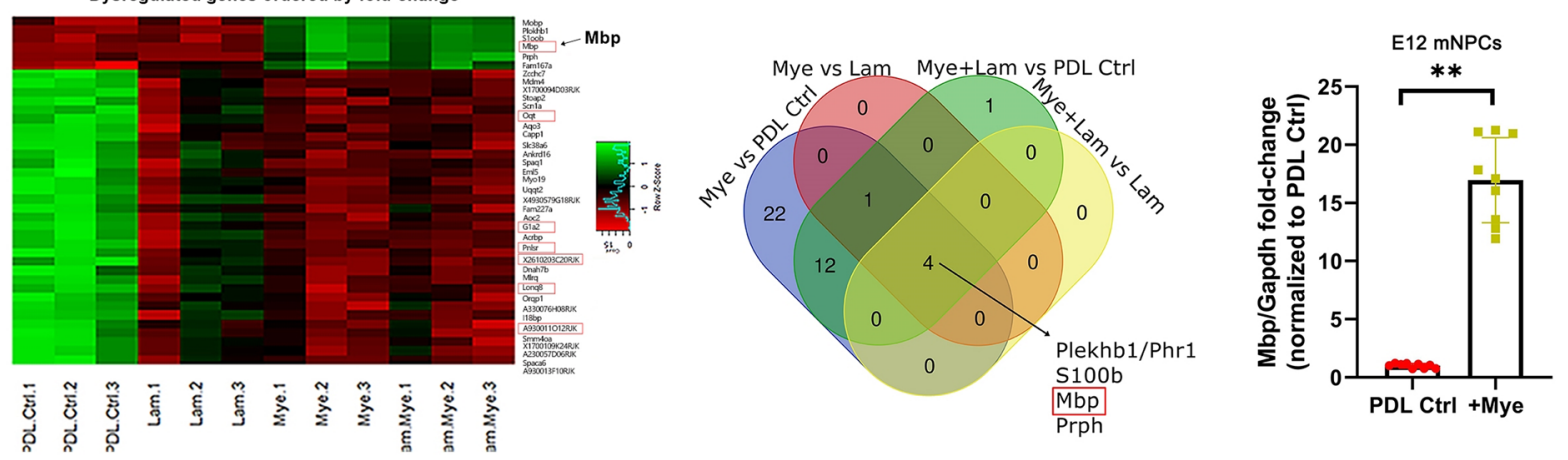

f

g

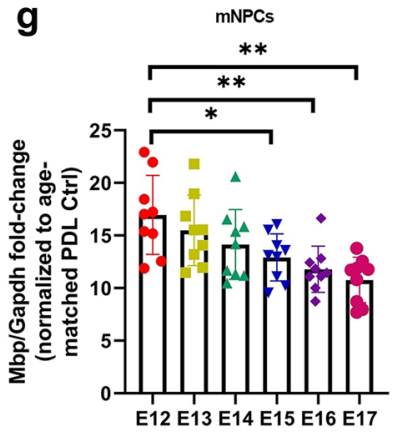

h

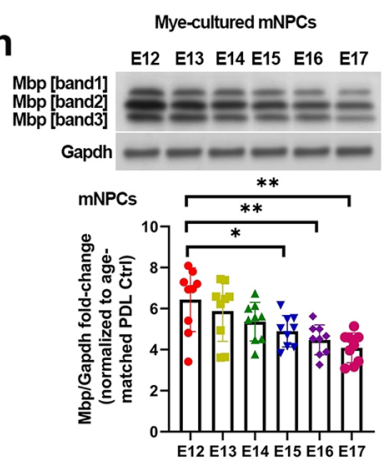

Fig. 1 R-based bioinformatics analysis of E12 mNPCs subjected to different substrates. R-based bioinformatics analysis of published RNA-seq data (GEO acc no. GSE98974) from spinal cord-derived E12 mouse neural progenitor cells (E12 mNPCs) that were cultured on Poly-d-lysine control (PDL Ctrl), laminin (Lam), myelin (Mye), or Mye+Lam substrate [false discovery rate (FDR) $<0.1, \mathrm{n}=3$ replicates/condition]. a Gene set enrichment analysis (GSEA)-based identification of six discreet gene co-expression modules (M1-6). Red coloring denotes a positive NES score, while blue coloring denotes a negative NES score. b The network plot and associated hub genes for the M6 gene co-expression module. $\mathbf{c}$ Heatmap of differentially-expressed genes (DEGs) ordered by descending log2 fold-change. Upregulation is denoted by green coloring, while downregulation is denoted by red coloring. e Venn diagram visualizing overlapping upregulated DEGs associated with Mye substrate. $\mathbf{f}$ qPCR of Mbp mRNA expression and $\mathbf{g}$ immunoblotting of Mbp in whole-cell lysates from E12 mNPCs on PDL Ctrl or myelin (Mye) substrates. Gapdh is the housekeeping and loading control. $\mathbf{h}$ qPCR of Mbp mRNA expression and $\mathbf{i}$ immunoblotting of Mbp protein expression in mNPCs reveals maturation-based Mbp downregulation on Mye substrate. All panels report means \pm standard deviations (SDs). $n=3$ embryos/genotype $\times 3$ wells/embryo. ${ }^{*} P<0.05$, ${ }^{*} P<0.01$ [e, f Student's t-test; $\mathbf{g}, \mathbf{h}$ one-way ANOVA, post-hoc Tukey's test] 


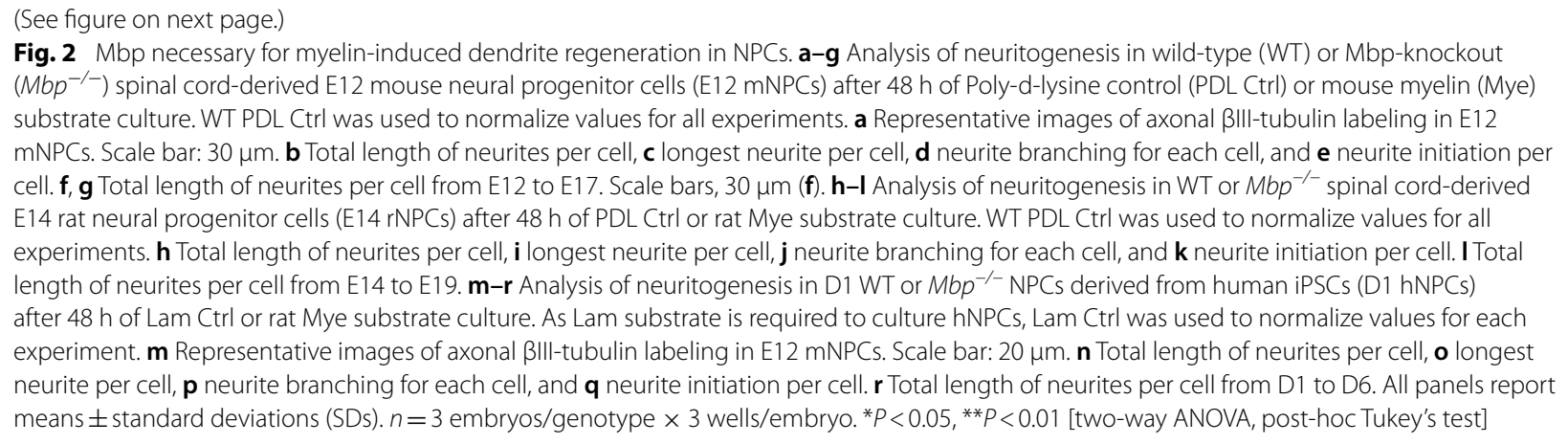

while no bands were detectable in $M b p$ knockout cells (Additional file 1: Fig. S3A-C). Neurite extensions were analyzed using anti-ßIII-tubulin antibody staining.

mNPCs displayed enhanced neurite lengths in the presence of Mye substrate (Fig. 2a-c). Mye substrate also stimulated greater neurite branching and initiation in mNPCs (Fig. 2d, e). Moreover, neurite outgrowth from mNPCs were dynamically measured from E12 to E17; Mye-induced outgrowth peaked at E12-13 and steadily declined thereafter (Fig. 2f, g). This finding was in agreement with the age-associated decline in Mbp expression (Fig. 1h, i). We found a similar pattern of findings in E14 rNPCs and D1 hNPCs cultured on rat Mye substrate (Fig. 2h-r). Notably, Mye-induced enhancements in neurite outgrowth and branching were abrogated by Mbp knockout in all three NPC models.

Mature neurons display myelin-based inhibition of neurite outgrowth in vitro [9]. If Mbp is essential to myelin-induced neurite outgrowth, Mbp overexpression may rescue myelin-based inhibition of neurite outgrowth in mature NPCs. Transfected E12 mNPCs were cultured on PDL Ctrl substrate for six days in order to induce maturation. The mature mNPCs were then re-plated on a myelin substrate. Mature mNPCs displayed myelin-based inhibition of neurite outgrowth, while Mbp overexpression partially rescued myelin-based inhibition (Additional file 1: Fig. S4A-E). We found a similar pattern of findings in E14 rNPCs and D1 hNPCs (Additional file 1: Fig. $\mathrm{S} 4 \mathrm{~F}-\mathrm{O})$. This data indicates that myelin promotes axonal outgrowth from NPCs in an Mbp-dependent manner.

\section{Mbp stimulates axonal outgrowth from NPCs via cleaving extracellular L1 cam to produce L1-70}

Mbp has been shown to be released into the extracellular space of adult mammalian neurons [10]. Mbp possesses serine protease activity and cleaves the extracellular protein L1 cell adhesion molecule (L1cam) to produce the $70-\mathrm{kDa}$ L1cam fragment (L1-70), which potentiates L1cam-dependent neurite outgrowth in adult mammalian neurons [10]. Therefore, we hypothesized that Mbp's serine protease activity would enhance extracellular L1cam cleavage and downstream neurite outgrowth in NPCs.

We first analyzed the localization of Mbp and L1cam in mNPCs by fluorescent immunostaining. Employing the pan-Mbp antibody and L1cam antibody-557 against mNPCs cultured on myelin substrate, we observed pronounced Mbp staining localized on the cell surface of WT mNPCs (Fig. 3a). We also observed co-localization of Mbp and L1cam at the cell surface of WT mNPCs. However, Mbp staining was absent in $M b p$ knockout mNPCs. These results indicate that Mbp and L1cam are extracellularly localized in NPCs.

Next, we investigated Mbp's proteolytic processing of L1cam in E12 mNPCs with or without CRISPR/ Cas9-mediated $M b p$ knockout cultured on the myelin substrate. Immunoblotting analysis using the L1cam antibody-557 revealed downregulated L1-70 and upregulated parental L1-200 by Mbp knockout (Fig. 3b). Also, the L1cam antibody 172-R directed against L1cam's intracellular domain confirmed that L1-70 was downregulated by $M b p$ knockout (Fig. 3b). We then assessed whether $M b p$ overexpression enhances L1-70 production in E12 mNPCs. Mbp overexpression enhanced L1-70 production and reduced parental L1-200 expression, while the serine protease inhibitor aprotinin abolished these effects (Fig. 3c). These results show that Mbp's serine protease activity promotes L1-70 production in NPCs.

To assess if L1-70 functions as a downstream intermediary for Mbp's neuritogenic effects, neurite outgrowth was analyzed in E12 mNPCs, E14 rNPCs, or D1 hNPCs with or without CRISPR/Cas9-mediated $M b p$ knockout combined with L1-70 rescue overexpression. The reduction in neurite outgrowth by $M b p$ knockout was rescued by addition of L1-70 overexpression in E12 mNPCs (Fig. 3d-g), E14 rNPCs (Fig. 3h-k), and D1 hNPCs (Fig. 3l-o). These results indicate that Mbp's stimulatory effects on neurite outgrowth in NPCs are mediated by 


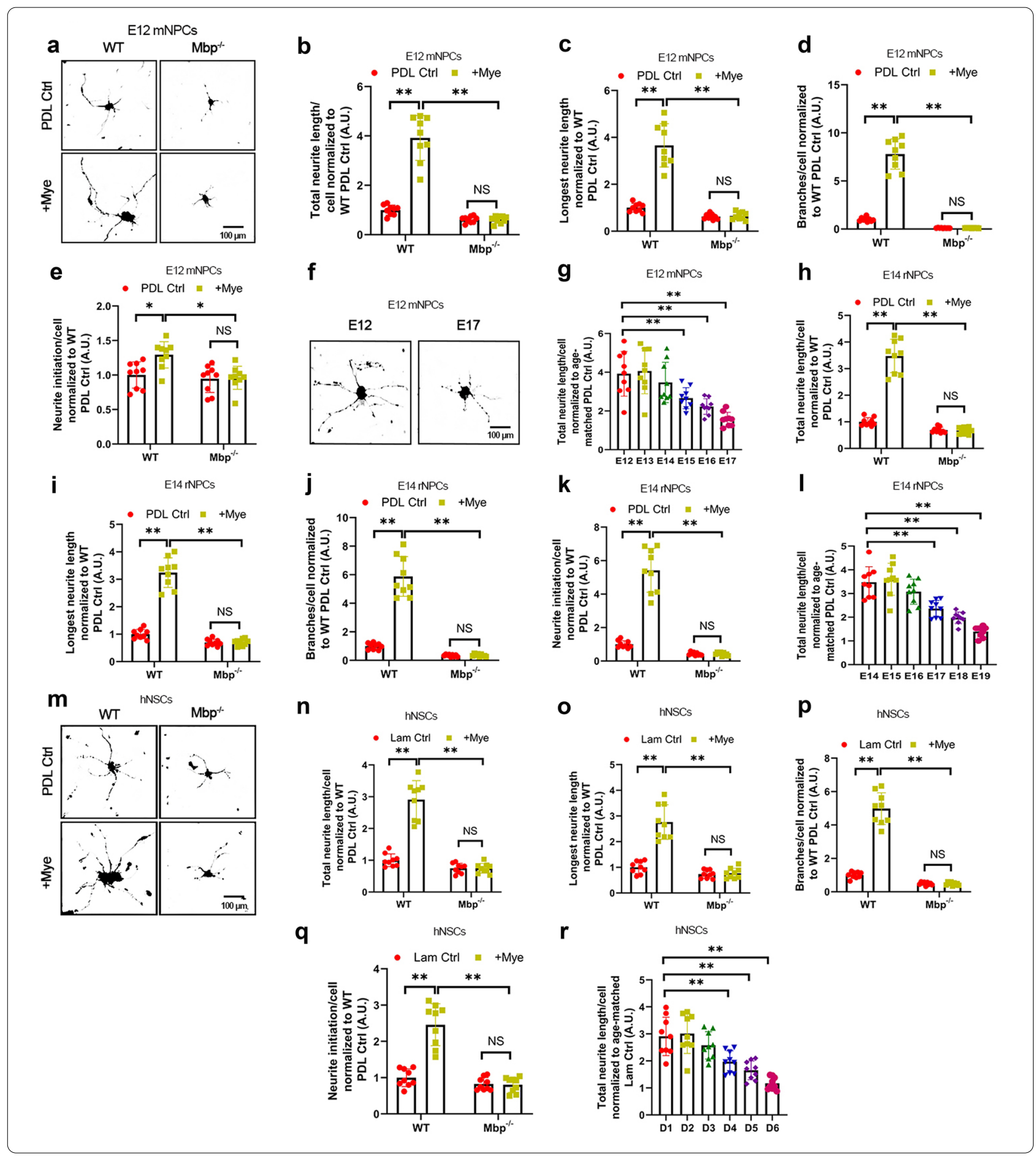




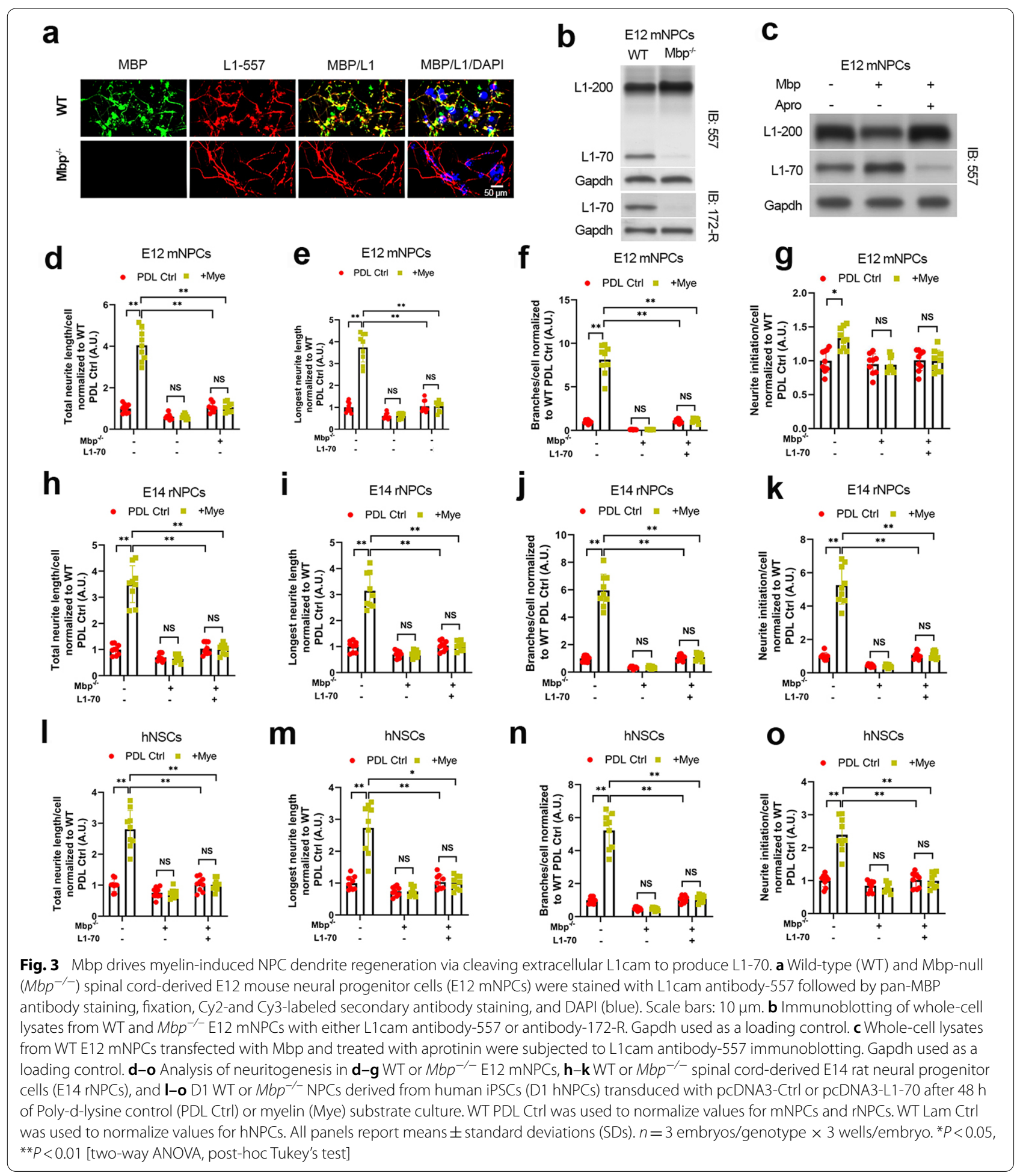




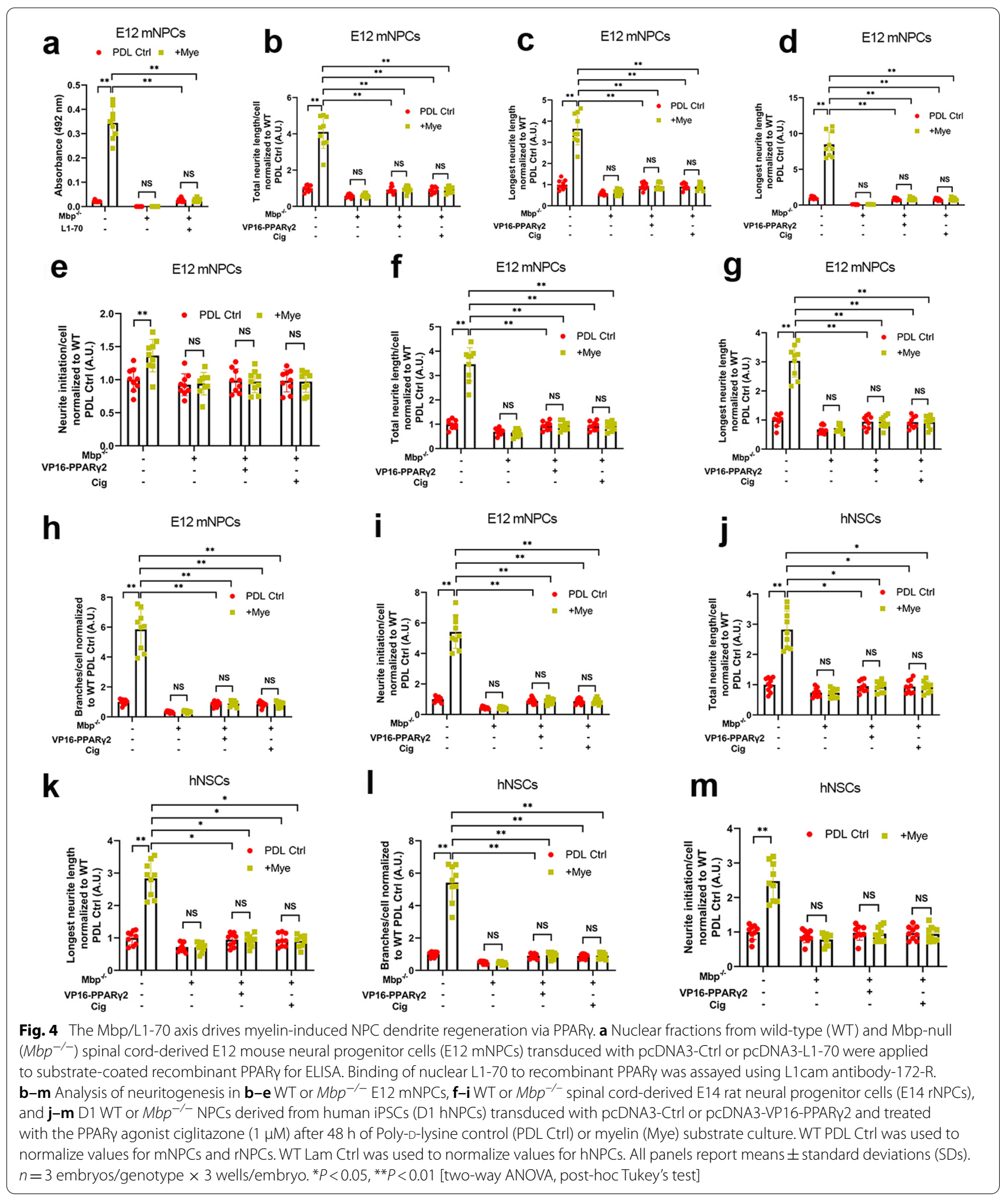


Mbp's production of L1-70 and establish the presence of a neuritogenic $\mathrm{Mbp} / \mathrm{L1}-70$ axis in NPCs.

\section{Mbp/L1-70 axis stimulates axonal outgrowth from NPCs via PPAR $\gamma$}

L1-70, which possesses the nuclear receptor co-activator motif LXXLL ( $\mathrm{L}_{1136} \mathrm{LILL}$ ), has been shown to translocate to the nucleus and bind to the nuclear receptor PPAR $\gamma$, thereby regulating PPAR $\gamma$ target gene transactivation in mouse neurons [11]. Moreover, the PPAR $\gamma$ agonists ciglitazone and pioglitazone promote neurite outgrowth in neuroblastoma cells $[12,13]$. This led us to hypothesize that the Mbp/L1-70 axis promotes axonal outgrowth from NPCs via stimulating PPAR $\gamma$ activation.

We first validated whether nuclear L1-70 binds to PPAR $\gamma$ in E12 mNPCs with or without CRISPR/Cas9mediated $M b p$ knockout cultured on the myelin substrate. Using ELISA, we applied the nuclear fraction from E12 mNPCs to substrate-coated recombinant PPAR $\gamma$. Nuclear fractions from WT E12 mNPCs showed strong binding of nuclear L1-70 to recombinant PPARY (Fig. 4a). However, the binding strength was attenuated by $M b p$ knockout and rescued by addition of L1-70 overexpression. These results indicate that Mbp's generation of L1-70 drives nuclear L1-70's binding to PPAR $\gamma$ in NPCs.

To assess if PPARy functions as a downstream intermediary for Mbp/L1-70's neuritogenic effects, neurite outgrowth was analyzed in E12 mNPCs, E14 rNPCs, or D1 hNPCs with or without CRISPR/Cas9-mediated Mbp knockout. The reduction in neurite outgrowth by $M b p$ knockout was rescued by constitutively-active VP16PPAR 2 or ciglitazone in E12 mNPCs (Fig. 4b-e), E14 rNPCs (Fig. 4f-i), and D1 hNPCs (Fig. 4j-m). These results indicate that $\mathrm{Mbp} / \mathrm{L} 1-70$ 's stimulatory effects on neurite outgrowth in NPCs are mediated by PPARy activity.

\section{Mbp/L1-70 axis regulates expression of PPARY's neuron differentiation-associated gene targets in NPCs}

Activated PPAR $y$ typically functions as a transcriptional repressor by binding to target gene promoters and reducing their transactivation [12]. Notably, three PPARY target genes are associated with neuron differentiation and are dysregulated in a rat dorsal root crush model, namely Apbb1, Cdkn1c, and Rtn4rl2 [12]. To assess if PPARY functions as a downstream repressor of these three target genes in NPCs, quantitative ChIP of Apbb1, Cdkn1c, and Rtn4rl2 was performed in E12 mNPCs with or without CRISPR/Cas9-mediated Mbp knockout. E12 mNPCs were stimulated with DMSO vehicle or ciglitazone; after $24 \mathrm{~h}$, chromatin complexes were cross-linked and subjected to anti-PPAR $\gamma$ ChIP. We found specific binding interactions between PPAR $\gamma$ and the Apbb1, Cdkn1c, and Rtn $4 r l 2$ gene promoters, which were downregulated by $M b p$ knockout and rescued by VP16-PPAR 2 or ciglitazone (Fig. $5 \mathrm{a}-\mathrm{C}$ ). qPCR analysis revealed that $M b p$ knockout significantly enhanced Apbb1, Cdkn1c, and Rtn4rl2 mRNA expression, which was rescued by VP16-PPAR 2 or ciglitazone (Fig. $5 \mathrm{~d}-\mathrm{f}$ ). These results demonstrate that the $\mathrm{Mbp} / \mathrm{L} 1-70$ axis inhibits PPAR $\gamma$-mediated repression of neuron differentiation-associated gene expression.

PPAR $\gamma$ activity stimulates Erk1/2 (p42/p44 MAPK) activation [13], which has been shown to enhance neurite outgrowth in NPCs [8]. This led us to hypothesize that the Mbp/L1-70/PPAR $\gamma$ axis may upregulate Erk1/2 activation in NPCs. Therefore, we investigated Erk1/2 activation in E12 mNPCs with or without CRISPR/Cas9mediated $M b p$ knockout. ELISA and immunoblotting at $6 \mathrm{~h}$ of substrate culture revealed that Erk1/2 phosphorylation was downregulated by $M b p$ knockout, which was rescued by VP16-PPAR 2 or ciglitazone (Fig. $5 \mathrm{~g}, \mathrm{~h}$ ). These results indicate that the Mbp/L1-70/PPARY axis promotes neuritogenic Erk1/2 activation in NPCs.

\section{Stable Mbp overexpression in scaffolded mNPCs improves locomotive recovery and axonal regeneration post-SCI} mNPCs seeded on porous collagen-based scaffolding (PCS) in a murine dorsal column crush model of SCI (characterized by irreversible hind limb instability and coordination dysfunction) have been shown to significantly improve axonal regeneration and locomotive recovery in vivo [9]. Based on Mbp's regenerative effects in vitro, we hypothesized that PCS-seeded mNPCs stably overexpressing $M b p$ would significantly enhance locomotive recovery in this murine model of SCI.

The in vivo experiments consisted of five cohorts of mice (i.e., Sham Ctrl, SCI Ctrl, SCI+Scaffold, $\mathrm{SCI}+$ Scaffold $+\mathrm{mNPC}$, and $\mathrm{SCI}+$ Scaffold $+\mathrm{Mbp}-\mathrm{mNPC}$ ), with eight mice per cohort (Fig. 6a). Post-mortem H\&E staining of SCI lesion sections at 6 weeks, 9 weeks, and 12 weeks post-SCI revealed that $\mathrm{mNPC}$-seeded and Mbp-mNPC-seeded scaffolds fully integrated into the surrounding nerve tissue (Fig. 6b). However, scaffoldonly grafts had migrated from the SCI lesion site in $100 \%$ of mice at 6 weeks post-SCI injury (Fig. 6b).

Locomotive recovery following SCI was analyzed via the locomotive fault rate on the horizontal ladder walking assay over the 12-week period post-SCI. The fault rate remained unchanged in the Sham Ctrl group over the 12-week evaluation period (Fig. 6c). However, over the 12-week evaluation period, we detected a significant effect of SCI $(P<0.0001$ [repeated measures two-way ANOVA], $F[4,480]=70.10)$. Starting at three weeks post$\mathrm{SCI}$, the locomotive performance in both $\mathrm{mNPC}$ groups 
a

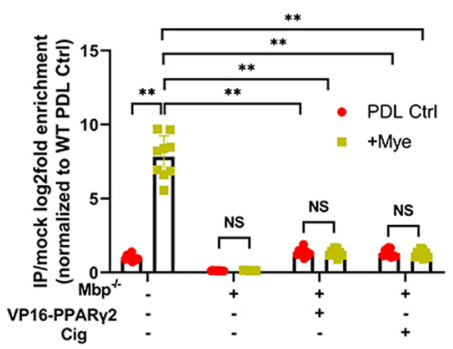

d

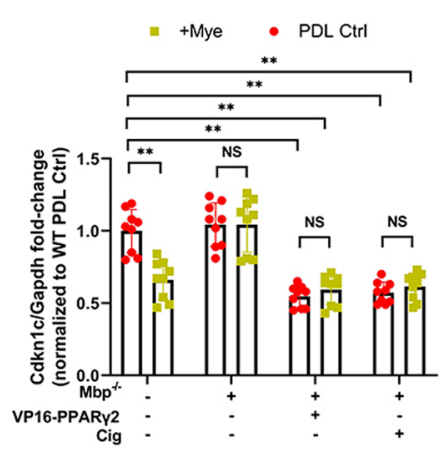

b

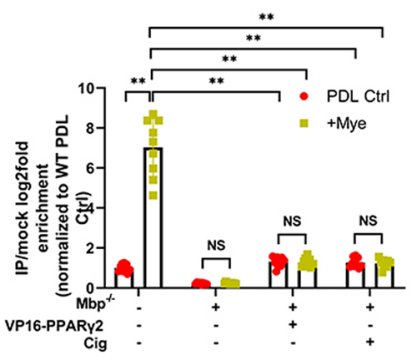

e

E12 mNPCs

- PDL Ctrl $\equiv+$ Mye

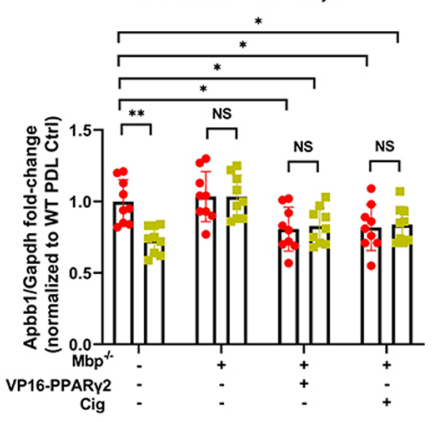

C

E12 mNPCs

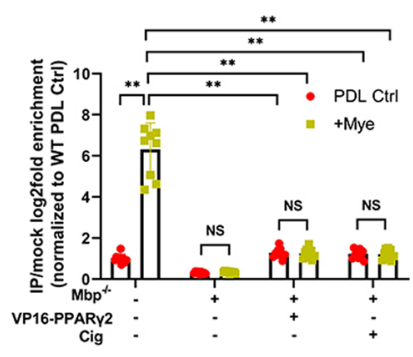

f

E12 mNPCs

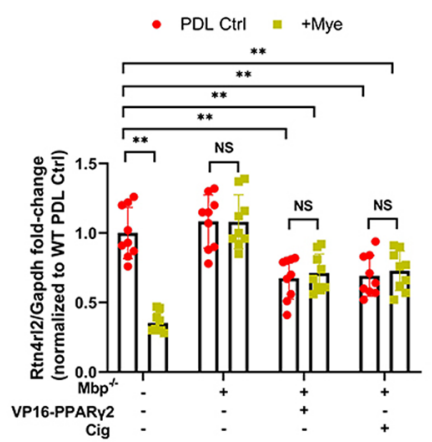

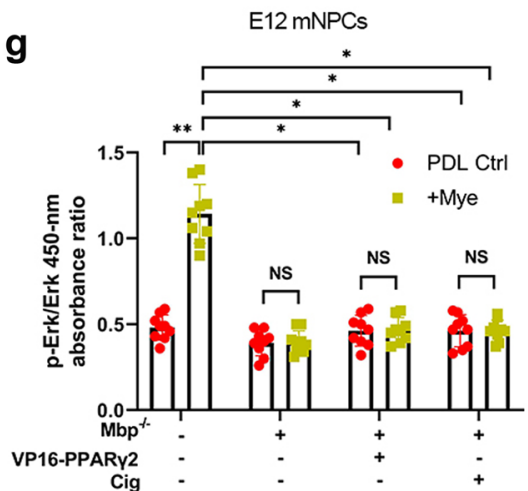

h

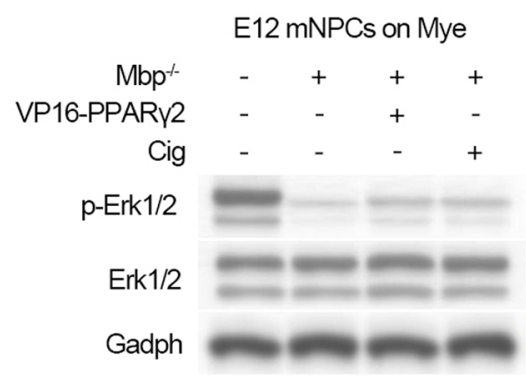

Fig. $5 \mathrm{Mbp} / \mathrm{L}$ 1-70 axis regulates expression of PPARY's dendrite regeneration-associated gene targets in NPCs. Wild-type (WT) and Mbp-null $\left(\mathrm{MbP}^{-1-}\right.$ ) spinal cord-derived E12 mouse neural progenitor cells (E12 mNPCs) transduced with pcDNA3-Ctrl or pcDNA3-VP16-PPARY2 and treated with the PPARy agonist ciglitazone $(1 \mu \mathrm{M})$ after $48 \mathrm{~h}$ of Poly-d-lysine control (PDL Ctrl) or myelin (Mye) substrate culture. WT PDL Ctrl was used to normalize values. a-c PCR quantification of anti-PPARy immunoprecipitated chromatin using site-specific primers. $\mathbf{d}-\mathbf{f}$ qPCR of PPAR target gene expression. $\mathbf{g}, \mathbf{h}$ Activation of extracellular signal-regulated kinase 1/2 (Erk1/2) signaling assayed by $\mathbf{g}$ ELISA and $\mathbf{h}$ immunoblotting. All panels report means \pm standard deviations (SDs). $n=3$ embryos/genotype $\times 3$ wells/embryo. ${ }^{*} P<0.05,{ }^{*} P<0.01$ [two-way ANOVA, post-hoc Tukey's test]

\section{(See figure on next page.)}

Fig. 6 Stable Mbp overexpression in scaffolded mNPCs produces superior locomotive recovery and axonal regeneration in a murine model of $\mathrm{SCl}$. a Experimental schematic overview of murine model of SCl detailing timepoints for spinal cord crush injury (SCl), grafting, locomotive fault analysis, and sacrifice for histological analysis. b H\&E-stained parasagittal spinal cord sections 6 weeks post-SCl. Scale bar, 200 Hm. c Quantification of fault rates by horizontal ladder walking assay post-SCl. $\mathbf{d}$ Boxplots of fault rates at 9, 10, 11, and 12 weeks post-SCl. e Representative immunofluorescent images of parasagittal spinal cord sections 6 weeks post-SCl stained for bovine collagen I, neurofilament heavy chain (NF), and L1-70. Scale bar, $200 \mu \mathrm{m}$. f Quantification of L1-70 + pixel density 9 weeks and 12 weeks post-SCI caudally, epicentrally, and rostrally to the SCI lesion. Results are normalized to the Sham Ctrl group. Panels report either means \pm standard deviations (SDs) [bar and line charts] or medians \pm interquartile ranges (IQRs) [boxplots]. $n=9$ animals per cohort. ${ }^{*} P<0.05,{ }^{*} P<0.01$ [one-way ANOVA, post-hoc Tukey's test] 
a

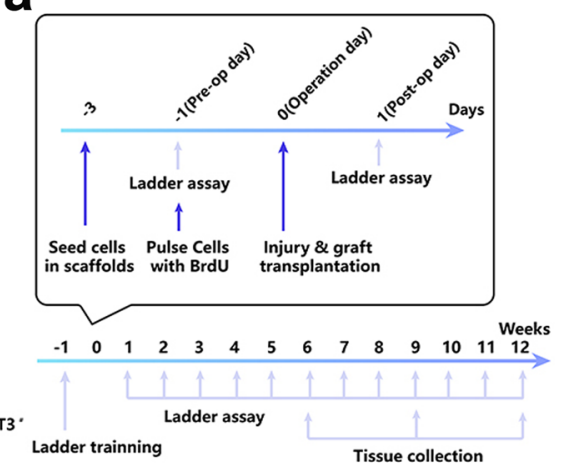

b

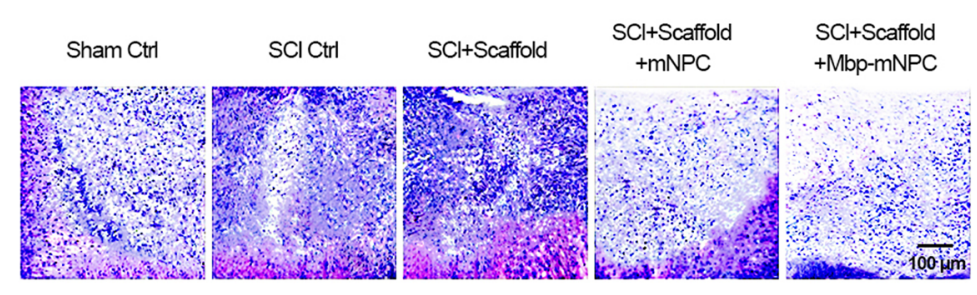

C

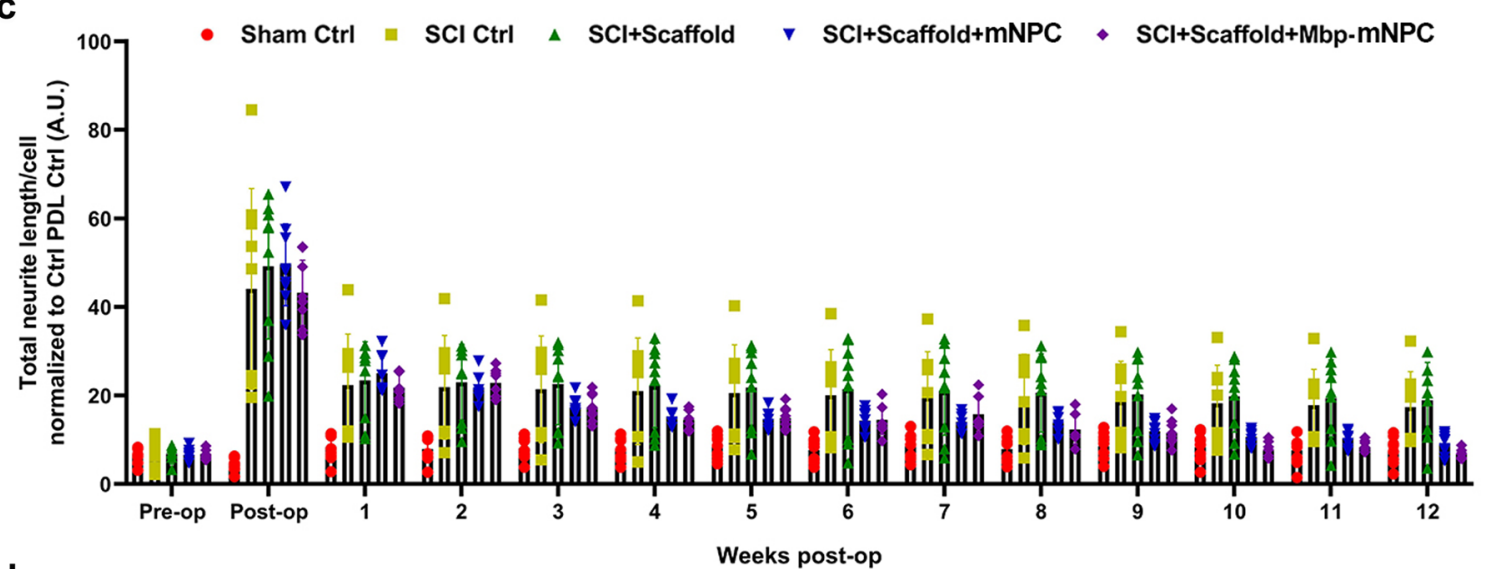

d

Week 9 post-op

Week 10 post-op

Week 11 post-op

Week 12 post-op
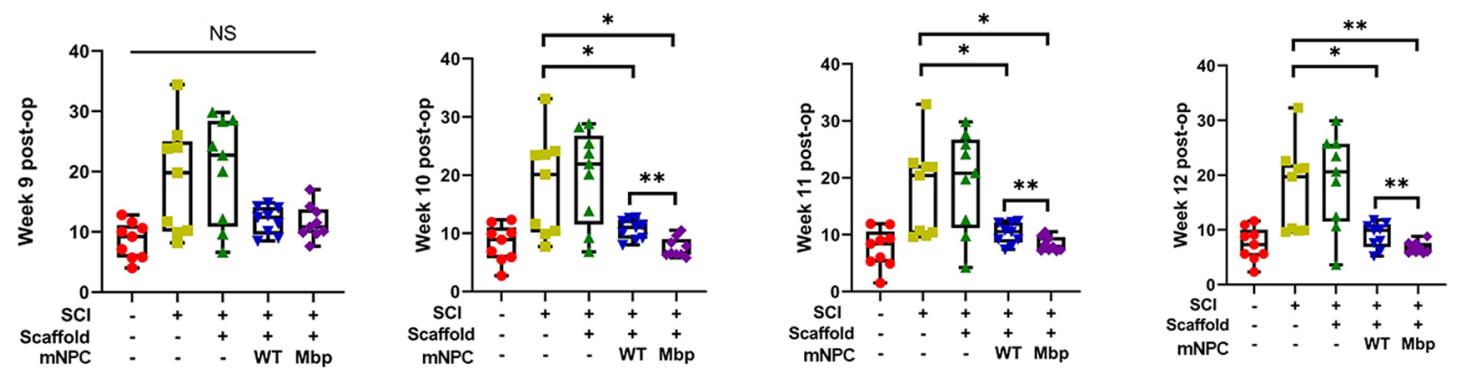

e

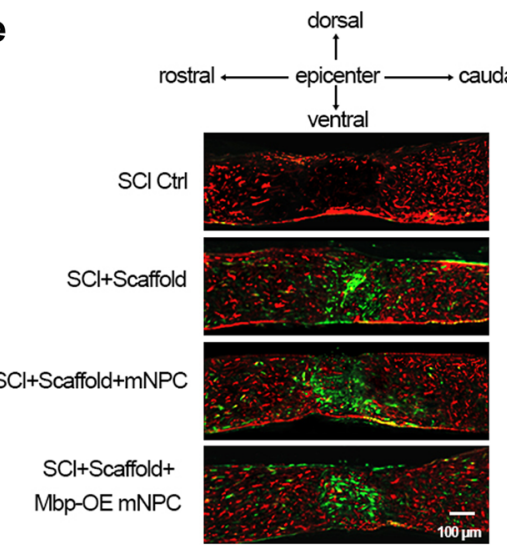

f

- $\mathrm{SCl}$ Ctrl = SCl+Scaffold - $\mathrm{SCl}+$ Scaffold $+\mathrm{mNPC}$

- SCl+Scaffold+Mbp-OE mNPC

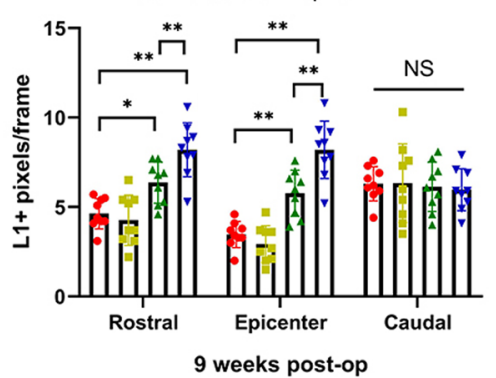

- $\mathrm{SCl} \mathrm{Ctrl}=\mathrm{SCl}+$ Scaffold - $\mathrm{SCl}+$ Scaffold + $\mathrm{mNPC}$

- SCl+Scaffold+Mbp-OE mNPC

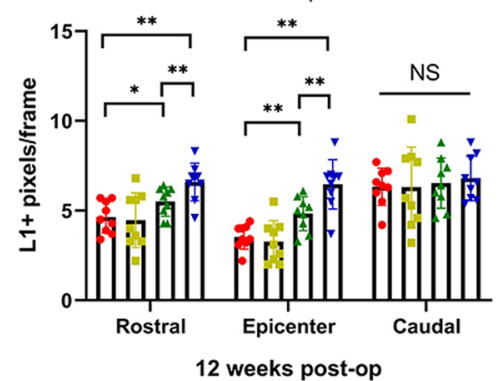


improved over the other SCI groups $(P<0.0001$ [repeated measures two-way ANOVA], F[3,384]=27.26). Notably, the SCI+Scaffold+Mbp-mNPC group showed a significant superiority over the $\mathrm{SCI}+\mathrm{Scaffold}+\mathrm{mNPC}$ group at ten weeks, eleven weeks, and twelve weeks post-SCI (Fig. 6d).

Axon regeneration through the SCI lesion is necessary for locomotive recovery [9]. Therefore, we analyzed axonal regeneration following SCI via NF and L1-70 immunostaining at nine and 12 weeks post-SCI. Nine weeks post-SCI, immunostaining of the SCI Ctrl sections revealed weak L1-70 staining at the SCI lesion site (Fig. 6e) In contrast, the mNPC groups revealed significant numbers of L1-70+axons at the lesion epicenter as well as rostrally and caudally of the SCI lesion site (Fig. 6e). At nine and 12 weeks post-SCI, both mNPC groups showed significantly greater L1-70 pixel density relative to the SCI Ctrl group rostrally and epicentrally, with the $\mathrm{SCI}+\mathrm{Scaffold}+\mathrm{Mbp}-\mathrm{mNPC}$ group showing significant superiority over the $\mathrm{SCI}+\mathrm{Scaffold}+\mathrm{mNPC}$ group (Fig. 6f). These results reveal that PCS-seeded mNPCs stably overexpressing $M b p$ significantly enhance locomotive recovery and axonal regeneration in this murine model of SCI.

\section{Discussion}

NPC-based regenerative therapies for SCI are aimed at stimulating NPC axons to grow into the host spinal cord tissue past the site of injury, thereby forming functional synaptic connections with the host neurons. Myelin-a multi-lamellar plasma membrane extension formed from oligodendrocytes that spirally wrap around the axon is a biologically-active substrate that supports axonal growth [14]. Groundbreaking work by Poplawski et al. has revealed that myelin augments neurite outgrowth from NPCs [8]. Here, we conducted an in silico bioinformatics analysis to identify key gene(s) that may participate in myelin-associated axonal regeneration from murine NPCs, which identified the serine protease Mbp. We also present in vitro and in vivo evidence that supports axonal regeneration from mammalian NPCs through the novel $\mathrm{Mbp} / \mathrm{L} 1 \mathrm{cam} / \mathrm{Ppar}$ signaling pathway.

In this study, $M b p$ was identified through bioinformatics analysis of published RNA-seq data (GEO accession no. GSE98974 [8]); follow-up validation through $M b p$ knockout/Mbp-overexpression studies revealed that $M b p$ mediates myelin-induced neurite outgrowth from NPCs in vitro and axonal regeneration in a murine model of SCI. Mbp-a positively-charged, disordered polypeptide is a key structural component of myelin that resides between the negatively-charged, cytoplasmic leaflets of the myelin membrane [14]. Mbp's interaction with these cytoplasmic leaflets triggers Mbp's polymeric self-assembly, thereby expelling cytoplasm from the myelin sheath's interior and promoting healthy membrane compaction [14]. In addition to this structural function, the 21.5$\mathrm{kDa}$ isoform of Mbp associates with the C-terminus of dynamin I to create a catalytically-active serine protease [15]. On the cell surface, this catalytically-active Mbp isoform cleaves the parental L1cam glycoprotein L1-200 at the arginine 687 residue (within the first FNIII domain of L1cam) to generate L1-70 (a transmembrane C-terminal fragment) and L1-135 [15] (an extracellular N-terminal fragment). Here, we found that Mbp's stimulatory effects on neurite outgrowth in NPCs are mediated by Mbp's production of L1-70. Indeed, several lines of published research support that Mbp-generated L1-70 promotes neuritogenesis and axonal regeneration. In vitro, Mbpgenerated L1-70 promotes neurite outgrowth in cerebellar neurons, which is abrogated by Mbp knockdown, blockade with an anti-Mbp antibody, or delivery of a L1cam peptide with a competitive Mbp cleavage site [10]. In mice, Mbp knockout or targeted mutagenesis of Mbp's proteolytically-active site eliminates L1-70 generation [10], while applying Mbp onto injured spinal cord tissue or viral-mediated Mbp overexpression in injured spinal cord tissue promotes L1-70 expression, re-myelination, and functional recovery post-SCI, while blockade with an anti-Mbp antibody or viral-mediated overexpression of proteolytically-inactive Mbp produces the opposite effects [16]. Moreover, the small-molecule L1cam mimic phenelzine upregulates spinal cord L1cam, improves hind limb functioning, and stimulates axonal regeneration at four and 5 weeks post-SCI in mice [17]. Furthermore, viral-mediated L1cam overexpression in a spinal cord transection (SCT) rat model reveals that post-SCI generation of diffusible L1cam fragments improves neurite outgrowth and myelination [18].

Delving further into Mbp/L1-70's mechanism of action, we found that Mbp/L1-70's stimulatory effects on neurite outgrowth in NPCs are mediated by PPAR $\gamma$ activity. Previous research has shown that L1-70 is imported into the nucleus, wherein its nuclear receptor co-activator motif LXXLL $\left(\mathrm{L}_{1136}\right.$ LILL) mediates its binding to various nuclear receptors, including PPAR $\gamma$, estrogen receptor (ER) $\alpha$ and $\beta$, and retinoid X receptor $\beta$ (RXR $\beta)$ [11]. In utero mutations in this LXXLL motif within murine embryos impairs cerebellar synaptic connectivity, motor coordination, and cognition in adulthood [11], suggesting that L1-70's interaction with these nuclear receptors regulates neural plasticity pathways. Indeed, we found that the Mbp/L1-70 axis inhibits PPAR $\gamma$-mediated repression of neuron differentiation-associated gene expression and promotes neuritogenic Erk1/2 activation in NPCs. 
There are several limitations to this study. First, although $M b p$ knockout produced profound neurite growth reductions in NPCs cultured on myelin, it is reasonable to assume that there may be other myelinassociated proteins that significantly impact neurite outgrowth. Second, we focused on Mbp/L1-70's stimulatory effects on PPAR $\gamma$ activity as a key regulator of neurite outgrowth from NPCs. It is possible that other downstream target(s) of the Mbp/L1-70 axis may have an impact on neurite outgrowth from NPCs. As a more complete profile of the molecular players involved in myelin-mediated NPC axonal regeneration would greatly aid therapeutic development for SCI, these issues require further evaluation.

In conclusion, we report that Mbp supports axonal regeneration from mammalian NPCs through the novel $\mathrm{Mbp} / \mathrm{L} 1 \mathrm{cam} / \mathrm{Ppar}$ signaling pathway. This study also indicates that bioengineered, NPC-based interventions can promote axonal regeneration and functional recovery post-SCI.

\section{Methods}

The detailed procedures regarding the R-based bioinformatics analysis, mice and rat subjects, myelin extraction, preparation and maintenance of cell culture plates, generation and culture of NPCs, plasmid construction and transfection, maturation assays, fluorescent immunostaining, quantitative polymerase chain reaction (qPCR), enzyme-linked immunosorbent assay (ELISA), immunoblotting, and the murine dorsal column crush model of SCI are provided in the Supplementary Methods. Unless stated otherwise, data are reported as means \pm standard deviations (SDs). A two-tailed, unpaired Student's $t$-test, one-way analysis of variance (ANOVA) with Tukey's post-hoc test, or two-way ANOVA with Tukey's post-hoc test (Additional file 1: Table S1) were employed for statistical comparisons between experimental groups as indicated. A repeated measures two-way ANOVA was used to analyze changes in locomotive recovery. All statistical analyses utilized a strict significance criterion $\left({ }^{*} P<0.05,{ }^{* *} P<0.01\right)$.

\footnotetext{
Abbreviations

CNS: Central nervous system; DEGs: Differentially-expressed genes; ER: Estrogen receptor; Erk1/2: Extracellular signal-regulated kinase; FDR: False discovery rate; IHC: Immunohistochemical; L1 cam: L1 cell adhesion molecule; Lam: Laminin; Mye: Myelin; Mbp: Myelin basic protein; MAG: Myelinassociated glycoprotein; NPCs: Neural progenitor cells; NF: Neurofilament; NES: Normalized enrichment scores; OMgp: Oligodendrocyte myelin glycoprotein; PBS: phosphate-bufferedsaline; PCS: porous collagen-based scaffolding; QPCR: Quantitative polymerase chain reaction; SCI: Spinal cord injury; WT: Wild-type.
}

\section{Supplementary Information}

The online version contains supplementary material available at https://doi. org/10.1186/s13578-021-00584-7.

Additional file 1. Supplementary Figures S1-S4.

Acknowledgements

Not applicable.

\section{Authors' contributions}

Conceived and designed the study: SC. Performed the experimental procedures: ZY, LC and LL. Analyzed the data: XJ. Drafted the manuscript: SC. All authors read and approved the final manuscript.

\section{Funding}

This work was supported by the National Natural Science Foundation of China (Grant No. 81700573). The funders had no role in study design, data collection and analysis, decision to publish, or preparation of the manuscript.

\section{Availability of data and materials}

The data used to support the findings of this study are available from the corresponding author upon request.

\section{Declarations}

Ethics approval and consent to participate

Approval for this study's protocols was provided in advance by the Ethics Committee of the Second Affiliated Hospital to Chongqing Medical University (no. 2020033, Chongqing, China). All animal experiments were conducted according to the National Institutes of Health $(\mathrm{NIH})$ Guide for the Care and Use of Laboratory Animals (Bethesda, MD).

\section{Consent for publication}

Not applicable.

\section{Competing interests}

The authors declare that they have no competing interests.

\section{Author details}

${ }^{1}$ Department of Orthopedics, the Second Affiliated Hospital of Chongqing Medical University, No. 76 Linjiang Road, Yuzhong District, Chongqing 400010, China. ${ }^{2}$ Department of Orthopedics, the First Affiliated Hospital of Chongqing Medical University, Chongqing, China.

Received: 19 February 2021 Accepted: 30 March 2021

Published online: 29 April 2021

\section{References}

1. Kumar R, Lim J, Mekary RA, Rattani A, Dewan MC, Sharif SY, OsorioFonseca E, Park KB. Traumatic spinal injury: global epidemiology and worldwide volume. World Neurosurg. 2018;113:e345-63.

2. Center NSCIS. Facts and figures at a glance. Birmingham: University of Alabama at Birmingham; 2016. p. 10.

3. Jin MC, Medress ZA, Azad TD, Doulames VM, Veeravagu A. Stem cell therapies for acute spinal cord injury in humans: a review. NeuroSurg Focus. 2019;46(3):E10.

4. Rosenzweig ES, Brock JH, Lu P, Kumamaru H, Salegio EA, Kadoya K, Weber $J \mathrm{~L}$, Liang JJ, Moseanko R, Hawbecker S. Restorative effects of human neural stem cell grafts on the primate spinal cord. Nat Med. 2018;24(4):484.

5. Kadoya K, Lu P, Nguyen K, Lee-Kubli C, Kumamaru H, Yao L, Knackert J, Poplawski G, Dulin JN, Strobl H. Spinal cord reconstitution with homologous neural grafts enables robust corticospinal regeneration. Nat Med. 2016;22(5):479

6. Lu P, Woodruff G, Wang Y, Graham L, Hunt M, Wu D, Boehle E, Ahmad R, Poplawski G, Brock J. Long-distance axonal growth from human induced pluripotent stem cells after spinal cord injury. Neuron. 2014;83(4):789-96. 
7. Lu P, Blesch A, Graham L, Wang Y, Samara R, Banos K, Haringer V, Havton L, Weishaupt N, Bennett D. Motor axonal regeneration after partial and complete spinal cord transection. J Neurosci. 2012;32(24):8208-18.

8. Poplawski GH, Lie R, Hunt M, Kumamaru H, Kawaguchi R, Lu P, Schäfer MK, Woodruff G, Robinson J, Canete P. Adult rat myelin enhances axonal outgrowth from neural stem cells. Sci Transl Med. 2018;10(442):eaal2563.

9. Kourgiantaki A, Tzeranis DS, Karali K, Georgelou K, Bampoula E, Psilodimitrakopoulos S, Yannas IV, Stratakis E, Sidiropoulou K, Charalampopoulos I. Neural stem cell delivery via porous collagen scaffolds promotes neuronal differentiation and locomotion recovery in spinal cord injury. NPJ Regener Med. 2020;5(1):1-14.

10. Lutz D, Loers G, Kleene R, Oezen I, Kataria H, Katagihallimath N, Braren I, Harauz G, Schachner M. Myelin basic protein cleaves cell adhesion molecule L1 and promotes neuritogenesis and cell survival. J Biol Chem. 2014;289(19):13503-18.

11. Kraus K, Kleene R, Henis M, Braren I, Kataria H, Sharaf A, Loers G, Schachner M, Lutz D. A fragment of adhesion molecule L1 binds to nuclear receptors to regulate synaptic plasticity and motor coordination. Mol Neurobiol. 2018;55(9):7164-78.

12. Geeven G, MacGillavry HD, Eggers R, Sassen MM, Verhaagen J, Smit AB, de Gunst MC, van Kesteren RE. LLM3D: a log-linear modeling-based method to predict functional gene regulatory interactions from genome-wide expression data. Nucleic Acids Res. 2011;39(13):5313-27.

13. Miglio G, Rattazzi L, Rosa AC, Fantozzi R. PPARy stimulation promotes neurite outgrowth in SH-SY5Y human neuroblastoma cells. Neurosci Lett. 2009;454(2):134-8.
14. Snaidero N, Velte C, Myllykoski M, Raasakka A, Ignatev A, Werner HB, Erwig MS, Möbius W, Kursula P, Nave K-A. Antagonistic functions of MBP and CNP establish cytosolic channels in CNS myelin. Cell Rep. 2017;18(2):314-23.

15. Kleene R, Lutz D, Loers G, Bork U, Borgmeyer U, Hermans-Borgmeyer I, Schachner M. Revisiting the proteolytic processing of cell adhesion molecule L1. J Neurochem. 2020. https://doi.org/10.1111/jnc.15201.

16. Lutz D, Kataria H, Kleene R, Loers G, Chaudhary H, Guseva D, Wu B, Jakovcevski I, Schachner M. Myelin basic protein cleaves cell adhesion molecule L1 and improves regeneration after injury. Mol Neurobiol. 2016;53(5):3360-76.

17. Li R, Sahu S, Schachner M. Phenelzine, a small organic compound mimicking the functions of cell adhesion molecule L1, promotes functional recovery after mouse spinal cord injury. Restor Neurol Neurosci. 2018;36(4):469-83.

18. Płatek R, Grycz K, Więckowska A, Czarkowska-Bauch J, Skup M. L1 Cell Adhesion Molecule overexpression down regulates phosphacan and up regulates structural plasticity-related genes rostral and caudal to the complete spinal cord transection. J Neurotrauma. 2020;37(3):534-54.

\section{Publisher's note}

Springer Nature remains neutral with regard to jurisdictional claims in published maps and institutional affiliations.
Ready to submit your research? Choose BMC and benefit from:

- fast, convenient online submission

- thorough peer review by experienced researchers in your field

- rapid publication on acceptance

- support for research data, including large and complex data types

- gold Open Access which fosters wider collaboration and increased citations

- maximum visibility for your research: over $100 \mathrm{M}$ website views per year

At BMC, research is always in progress.

Learn more biomedcentral.com/submissions 